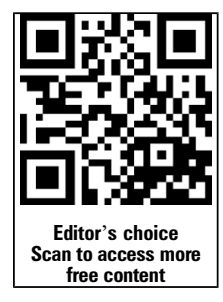

\title{
Incidence and morbidity of craniocervical arterial dissections in atraumatic subarachnoid hemorrhage patients who underwent aneurysmal repair
}

\author{
Kevin Carr, ${ }^{1}$ Fred Rincon, ${ }^{2,3}$ Mitchell Maltenfort, ${ }^{4}$ Lee Birnbaum, ${ }^{1,5}$ Bradley Dengler, ${ }^{1}$ \\ Michelle Rodriguez, ${ }^{6}$ Ali Seifi $^{1}$
}

${ }^{1}$ Department of Neurosurgery, University of Texas Health Science Center at San Antonio, San Antonio, Texas, USA ${ }^{2}$ Department of Neurology, Thomas Jefferson University, Philadelphia, Pennsylvania, USA

${ }^{3}$ Department of Neurosurgery, Thomas Jefferson University, Philadelphia, Pennsylvania, USA

${ }^{4}$ Rothman Institute, Philadelphia, Pennsylvania, USA

${ }^{5}$ Department of Neurology, University of Texas Health Science Center at San Antonio, San Antonio, Texas, USA

${ }^{6}$ School of Medicine, University of Texas Health Sciences Center, San Antonio, Texas, USA

\section{Correspondence to} Dr A Seifi, Department of Neurosurgery, Mail Code 7843, Medical School Building 102F, San Antonio, TX 78229-3900, USA; seifi@uthscsa.edu

Received 2 July 2014 Revised 21 July 2014 Accepted 23 July 2014 Published Online First 8 August 2014

\section{ABSTRACT}

Background No studies have assessed the incidence of craniocervical arterial dissections (CCADs) and its association to mortality in hospitalized patients with a primary diagnosis of atraumatic subarachnoid hemorrhage (SAH) requiring aneurysmal repair. We hypothesize that the incidence of CCADs in these patients has increased over time as well as its association to mortality.

Methods We conducted a 9 year retrospective assessment of the incidence of CCADs in patients hospitalized with a primary diagnosis of an $\mathrm{SAH}$ requiring repair and the effect of CCAD on mortality. Using the Nationwide Inpatient Sample (NIS), we queried records from 2003 to 2011 for an ICD-9 (International Classification of Diseases-9) code corresponding to admissions for atraumatic SAH. Demographical data, incidence of CCADs, type of aneurysmal repair, length of hospital stay, and hospital mortality were recorded. Multivariate logistical regression models were fitted to assess for the impact of CCAD on inhospital mortality and morbidity.

Results During the period 2003-2011, of the NIS reported 18260 patients who required aneurysmal SAH repair, 9737 (53.32\%) underwent endovascular coiling and $8523(46.48 \%)$ had surgical clipping. There were 131 patients in the cohort with reported CCADs: 94 $(71.75 \%)$ of these patients had received endovascular coiling repair and 37 (28.25\%) had undergone surgical clipping repair. Patients who underwent endovascular coiling had a higher rate of CCADs in this cohort (OR 2.94; $95 \% \mathrm{Cl} 2.00$ to $4.31, \mathrm{p}<0.0001$ ). The incidence of CCADs in this population increased by an average rate of $9.4 \%$ per year (OR 1.14; $95 \% \mathrm{Cl} 1.06$ to $1.23, p<0.0006$ ), from $0.49 \%$ in 2003 to $1.10 \%$ in 2011. The diagnosis of CCAD added 3 and 6 more days to median length of hospitalization stay for surgical clipping and endovascular coiling, respectively. The unadjusted rate of mortality was $8.4 \%$ in the CCADs subgroup, and the presence of CCAD was not a predictor of mortality in our multivariate regression model (OR 0.68; $95 \%$ Cl 0.36 to 1.27, $p=0.2244$ ).

Conclusions Our study indicates an annual increase in the incidence of CCADs in patients admitted with SAH who require aneurysmal repair. More than two-thirds of these patients that developed CCADs had undergone endovascular coiling repair. A diagnosis of CCAD increased the length of hospital stay but had no statistically significant association with mortality in this patient population.

\section{INTRODUCTION}

Craniocervical arterial dissections (CCADs) can occur from traumatic neck injury, ${ }^{1}$ spontaneously, ${ }^{2-4}$ secondary to underlying aneurysms, ${ }^{5}$ or as a complication of endovascular intervention. ${ }^{67}$ Small studies have demonstrated a relatively benign natural history for CCADs from any etiology. ${ }^{7}$ However, there is very little literature that has specifically assessed the incidence and morbidity of CCADs in the population of patients with atraumatic subarachnoid hemorrhage (SAH) requiring aneurysmal repair. ${ }^{9-12}$

The standard of care for patients with suspected aneurysmal SAH generally involves diagnosis using digital subtraction angiography followed by securing the aneurysm in suitable candidates with either surgical clipping or endovascular coiling. ${ }^{13}$ Modification of the Seldinger technique to access the cranial vasculature carries a risk of CCAD, ${ }^{14-16}$ which may impact morbidity and mortality.

In this study, we used the Nationwide Inpatient Survey (NIS) to define the incidence of CCADs in patients admitted with a primary diagnosis of $\mathrm{SAH}$ and requiring aneurysmal repair, as well as the associations between CCADs and treatment metrics, such as mortality and hospital length of stay (LOS). We also examined whether a significant difference exists between the incidences of CCADs in SAH patients who underwent endovascular coiling repair compared with surgical clipping.

\section{METHODS}

\section{Data selection and outcome variables}

The NIS databases were obtained from the Agency for Healthcare Research and Quality's Healthcare Cost and Utilization Project. This database represents approximately $20 \%$ of patient admissions every year at non-federal hospitals. Results were investigated from 2003 to 2011, the last year with available data.

Hospitalization patient records were selected from the NIS database using the Ninth International Classification of Diseases Clinical Modification Codes (ICD-9-CM) for atraumatic SAH (430) along with procedure codes for aneurysmal surgical clipping (39.51) and endovascular coiling $(39.79,39.72$, or 39.52$)$. Selection of these aneurysm codes has been previously validated. ${ }^{17-19}$ CCADs were identified based on ICD-9 codes 443.21 or 443.24 . 
Also collected were patient demographics (age, sex, race, primary payer, year of surgery) and hospital characteristics (region, size, urban academic vs urban private vs urban rural). The endpoints of interest were inhospital death and hospital LOS. Patients younger than 18 years were excluded. This analysis was exempt from full review by the institutional review board.

\section{Statistical analysis}

Logistic regression analyses were used to identify factors associated with in-hospital death and occurrence of CCAD. A negative binomial regression with a linear link was used to identify factors predictive of LOS. Statistical analysis was performed using the 'rms' and 'MASS' packages in the R programming language for statistical computing (V.3.0.1), both available under the GNU Public License (http://www.cran.r-project.org). For all regressions, a full model was created with all potential relevant predictors. The model was then pruned systematically using the Akaike Information Criterion, a criterion based on combined predictive power and complexity of the model, until no further pruning improved the Akaike Information Criterion.

\section{RESULTS \\ Demographics}

Mean age of patients in the endovascular coiling treatment group was 55.29 year (mean \pm SD, $55.29 \pm 14.45$ years) and 53.10 years in the surgical clipping group (53.10 \pm 12.98 years). In both treatment groups, women represented the majority of patients $(67.67 \%$ and $68.90 \%$ in the endovascular and clipping groups, respectively). Considering ethnicity, black and hispanic patients underwent more surgical clipping, while white and Asian patients had similar rates of endovascular coiling and surgical clipping. Data on demographics, insurance type, geographical region, hospital location, and size are compared in both subgroups in table 1.

\section{Incidence and epidemiology}

During the period 2003-2011, the NIS reported 18260 patients who required aneurysmal repair, of whom 9737 (53.32\%) underwent endovascular coiling and 8523 (46.48\%) required surgical clipping. During the period under study, there was generally a decreasing trend in the decision to treat surgically via open craniotomy and clipping; $22.78 \%$ in 2003, $11.53 \%$ in 2011. Conversely, the incidence of endovascular therapy in this patient cohort increased during this period; from $10.17 \%$ in 2003 to $17.42 \%$ in 2008 . After 2008 there was a general decrease in endovascular interventions (figure 1). There were 131 patients with reported CCADs, resulting in a cumulative prevalence of $0.72 \%$ over the course of the study. The incidence of CCADs in this population increased at a rate of $9.4 \%$ per year (OR 1.14; 95\% CI 1.06 to $1.23, \mathrm{p}<0.0006$ ), from $0.49 \%$ in 2003 to $1.10 \%$ in 2011 (figure 2).

Ninety-four $(71.75 \%)$ of these CCADs occurred in patients who received endovascular coiling and 37 (28.25\%) received surgical clipping. Patients who underwent endovascular coiling had a significantly higher rate of CCADs (OR 2.94; 95\% CI 2.00 to $4.31, \mathrm{p}<0.0001$ ) both in bivariate and multivariate analysis (figure 2).

As it related to LOS, a diagnosis of a CCAD increased median LOS by 3 and 6 days in the surgical clipping and endovascular treatment groups, respectively (figure 4).
Table 1 Characteristics of the study cohort

\begin{tabular}{lll}
\hline & Surgical clipping & Endovascular coiling \\
\hline No of patients & 8523 & 9737 \\
Age (years) (mean \pm SD) & $53.10( \pm 12.98)$ & $55.29( \pm 14.45)$ \\
Women $(\%)$ & 68.90 & 67.67 \\
White & 4317 & 4014 \\
Black & 1215 & 921 \\
Hispanic & 1122 & 798 \\
Asian & 291 & 264 \\
Native America & 34 & 22 \\
Other & 342 & 226 \\
Unspecified & 2416 & 2278 \\
Insurance & & \\
Medicare & 1915 & 2242 \\
Medicaid & 1513 & 1152 \\
Private & 4605 & 3855 \\
Self-pay & 1116 & 778 \\
No charge & 103 & 111 \\
Other & 468 & 358 \\
Geography & & \\
NE & 1573 & 1513 \\
Midwest & 1993 & 1787 \\
South & 3836 & 3267 \\
West & 2335 & 1956 \\
Rural & 152 & 80 \\
Urban academic & 1378 & 906 \\
Urban private & 8088 & 7463 \\
Hospital size & & \\
Small & 298 & 977 \\
Medium & 1443 & \\
Large & 7877 & \\
\hline & & \\
\hline
\end{tabular}

\section{Mortality}

During the study period, in the entire cohort, there was a general downward trend in mortality (OR 0.96; 95\% CI 0.95 to $0.98, \mathrm{p}<0.0001)$. The overall unadjusted mortality rate was $8.4 \%$ in the CCAD subgroup. Controlling for changing annual trends, patient age, and surgery type, the presence of CCADs was not associated with increased mortality in our multivariate

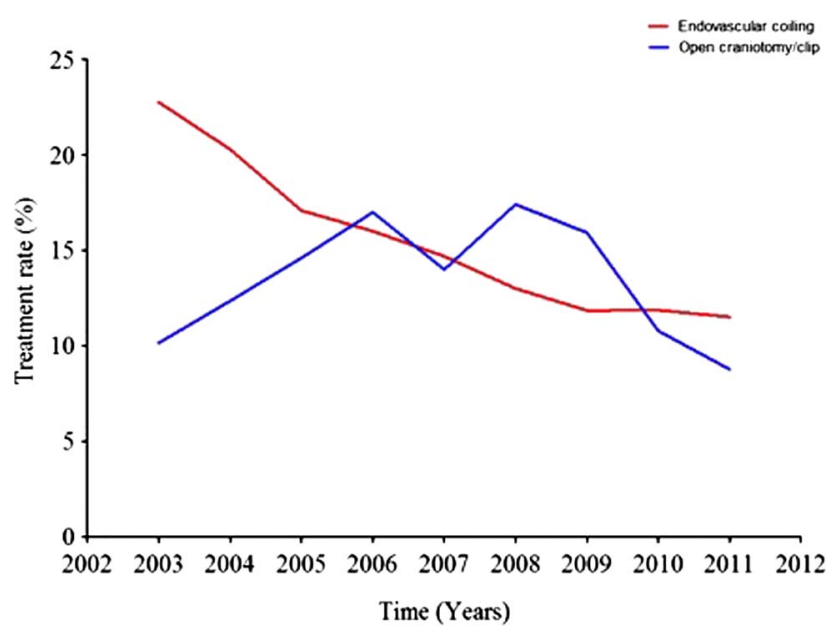

Figure 1 Incidence of the use of treatment modalities (endovascular coiling, surgical clipping) as a fraction of subarachnoid hemorrhage all comers, 2003-2011. 


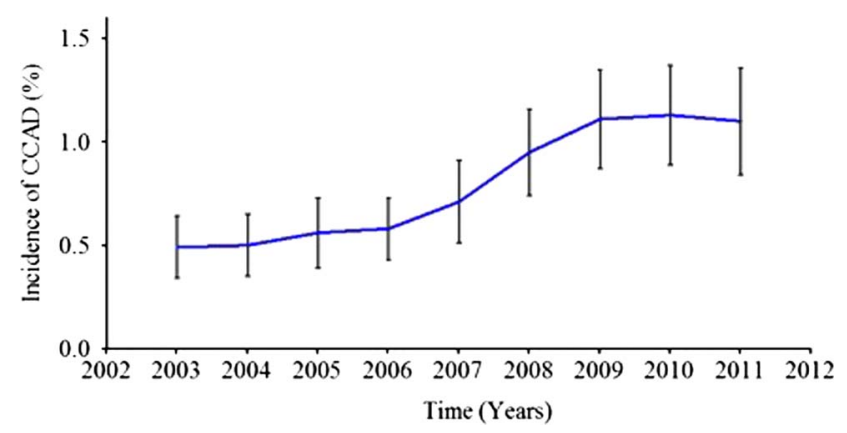

Figure 2 Incidence of craniocervical arterial dissections (CCADs) in the study cohort.

regression model (OR 0.68 ; 95\% CI 0.36 to $1.27, \mathrm{p}=0.2244$ ). Compared with the open craniotomy treatment group, endovascular coiling had a marginally greater association with mortality (OR 1.17 ; 95\% CI 1.07 to 1.27 ). The annual OR for mortality was found to be 1.03 (95\% CI 1.03 to $1.03, \mathrm{p}<0.0001)$, approximating to 1.34 per decade (95\% CI 1.30 to 1.38 , $\mathrm{p}<0.0001)$. The treatment subgroup that underwent endovascular coiling had higher mortality rates during the study period (OR 1.17; 95\% CI 1.07 to 1.27).

\section{DISCUSSION}

The rate of CCADs in patients who were treated for atraumatic SAH increased gradually from 2003 to 2011 (figure 2), and CCADs were more frequent in patients who required coiling compared with clipping during the study period (figure 3). While the presence of CCADs were not associated to mortality, it was associated with an increase in LOS in multivariate regression analyses (figure 4).

To assess the utilization of treatment modalities throughout the course of the study, we individually calculated the incidence of treatment utilization throughout the course of the study. Data from the NIS also demonstrated an increase in the rate of endovascular coiling from 2003 to 2006, with a decline beginning in 2008 (figure 1). We approximate that the indications for the various treatment modalities has remained relatively unchanged during this period. Previous studies examining data up to 2008 also indicated increased use for the treatment of ruptured and unruptured intracranial aneurysms. ${ }^{20-22}$ Our observation of declining rates of endovascular coiling from 2008 onward may be indicative of increased utilization of alternative endovascular

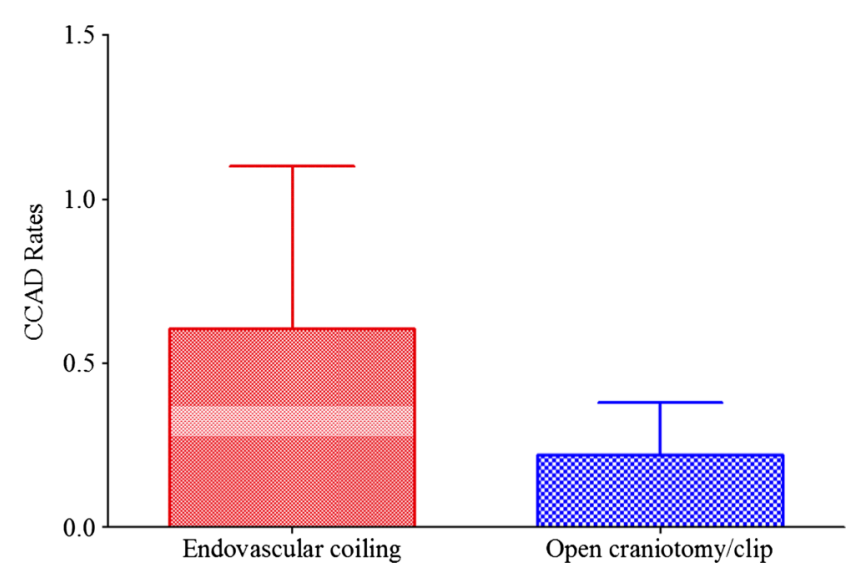

Figure 3 Cumulative prevalence of craniocervical arterial dissections (CCADs) in the surgical clipping and endovascular coiling groups.

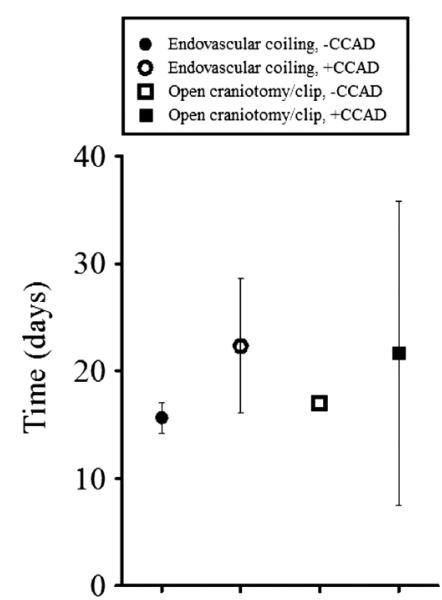

Figure 4 Impact of craniocervical arterial dissections (CCADs) on length of stay in the surgical clipping and endovascular coiling groups.

therapies, such as low porosity stenting, which may have been captured using separate ICD-9-CM codes.

During the endovascular treatment of aneurysmal SAHs, there is an associated risk of catheter related CCADs, pseudoaneurysms, and other arterio-occlusive phenomena. ${ }^{23} 24$ In patients requiring both diagnostic and interventional procedures, this risk increases. Results from our study were concordant with this fact as we demonstrated a statistically significant association between endovascular therapy and an increase in the diagnosis of CCADs (figure 3). Various studies have demonstrated that the incidence of iatrogenic CCADs after neurointerventional procedures is $0.1-0.6 \% .^{6}{ }^{25-27}$ These cases are often only detected after ischemia related neurological decline, or by imaging if clinical suspicion exists. ${ }^{6}$ As this was an observational study, we cannot assess causality between decision to intervene, type of intervention, and the occurrence of CCADs in this population. It is possible, however, that the presence of iatrogenic CCADs may explain the increased incidence in the endovascular treatment group compared with the open craniotomy group. Nonetheless, further prospective cohort analysis will be able to assess this relationship.

In our analysis, we demonstrated a nominal but statistically significant increased odds ratio of mortality in the treatment subgroup who required endovascular coiling therapy compared with the open craniotomy and clipping subgroup. While we cannot assess a causal relationship between our observations and intervention type, we do not suspect that there is a direct relationship. It is likely that a decision to intervene in cases that are amenable to both procedures may be based on the clinical stability of the patient. It is possible that patients who are thought to be less clinically stable, or with multiple medical comorbidities, may be biased towards the endovascular treatment subgroup. Kaku et $a l^{28}$ in their treatise posited that the overall clinical gestalt major factor impacting the choice to choose a 'safer treatment' or one that provides 'decades of cure'. Similarly, Darsaut et ${ } l^{29}$ suggested that 'poor medical condition' is a factor that is considered important in the decision between treatment modalities. Inevitably, patients' poor medical conditions may be an important factor associated with the relatively increased odds ratio of mortality.

Aneurysms of the carotid artery can be classified based on their morphology and underlying anatomical aberrances. ${ }^{5}$ In a seminal paper on the classification of non-atherosclerotic dissecting aneurysms, Mizutani et al proposed that the type 1 
classic dissecting aneurysm is most associated with SAH, and biomechanically presents with significant disruption of the internal elastic lamina. For these patients, a common treatment option is endovascular coiling, ${ }^{30}{ }^{31}$ which could also explain the association between the increased incidence of CCADs in the endovascular treatment group.

While the occurrence of iatrogenic CCADs is well described, various studies have shown good clinical outcome for patients treated via the endovascular approach. In a prospective assessment of intracranial dissections treated using the endovascular approach, Mohammadian et al demonstrated excellent return of function at long term follow-up in patients who received endovascular coiling or stenting. ${ }^{32}$ Not surprisingly, in their series, $83 \%$ of patients co-presented with $\mathrm{SAH}$, and clinical improvement was correlated to the extent of sentinel injury characterized by the initial modified Rankin Scale score. While statistical analysis did not characterize the extent of this association, patients without SAH had full return of function without deficits.

For patients with diagnosed CCADs, the treatment is usually endovascular stenting, anticoagulation and/or antiplatelet therapy, or a combination of both. ${ }^{8}{ }^{33-38}$ Although there are no randomized controlled trials assessing its utility in the treatment of CCADs to date, antiplatelet therapy is employed by many clinicians as part of their treatment strategy based on the recommendations of the Cervical Artery Dissection in Ischemic Stroke Study Groups. ${ }^{39}$ At our center, common practice is close observation of treated patients for several days in the event of aggressive anticoagulation or complications related to therapy. An increased hospital stay is generally required while transitioning to long term anticoagulation for non-occlusive dissections. If this is the general treatment strategy of clinicians nationally, then the increase in reported LOS for these patients observed in our analysis is explained.

In general, studies on the associations between CCADs and patient mortality have been sparse. The paucity of studies on $\mathrm{CCADs}^{7}$ may be explained by a relatively asymptomatic clinical course. In most cases, CCADs would be identifiable only by radiography, which would only be performed when it is clinically suspected. ${ }^{40}$ We have reported that the cumulative prevalence of CCADs over the course of the study was $0.72 \%$. Several single institutions reported CCAD incidence rates of $0.14-0.5 \%{ }^{6}{ }^{7} 23$ In 2000, Cloft et al demonstrated a $0.4 \%$ incidence of arterial dissections in a cohort of 3112 patients receiving neuroradiologic procedures (diagnostic cerebral angiogram, neurointerventional therapy). ${ }^{7}$ In subset analyses of patients receiving only neurointerventional therapies, this incidence increased to $0.7 \%$ but was not statistically significant. Of the 12 patients who developed CCADs, one patient died within $48 \mathrm{~h}$ and six patients were treated with antiplatelet or anticoagulant therapy for $24-48 \mathrm{~h}$. A later retrospective analysis of over 3000 patients receiving diagnostic angiography suggested an incidence of $0.14 \%$ (five patients). ${ }^{23}$ We observed an increased incidence of CCADs over the course of the study. Due to the limitations of the NIS database, whether or not this increase in incidence is related to an increase in detection due to improved technology, or is associated with increased utilization of endovascular techniques, is not known.

Fifi et al, in their study, reported a distinct age association, with older patients demonstrating an increased proclivity for developing this complication $(p=0.03) .{ }^{23}$ There was only one reported subacute stroke and one internal carotid artery dissection requiring stent placement for reperfusion. While the overall risk decreased with time over the course of the study, this was not statistically significant. The indications for intervention in their study were varied, with approximately $20 \%$ of the cohort presenting with a primary diagnosis of SAH and $26.9 \%$ with unruptured aneurysms. Of the five patients developing CCADs, two patients had a reported diagnosis of SAH. No patient admitted for unruptured aneurysms developed a CCAD. There were no reported data on the impact of these complications on length of hospitalization.

Similar to other studies, we demonstrated a relative benign course in patients who developed CCADs. Although an increasing incidence of CCADs was not associated with increased mortality, there was an associated increase in LOS in patients diagnosed with CCADs, and this increase was more prominent in the endovascular coil treatment group.

\section{Limitations}

This study has limitations that implicitly affect its interpretation. The diagnoses of SAH and CCADs are dependent on either clinical suspicion or standardized diagnosis protocols. Thus variability in practice throughout the period of time examined here has inevitably affected diagnosis and reporting. Additionally, increased awareness of the complication over time will also increase its reporting rates. Hospital discharge information does not differentiate between etiologies of reported CCADs. Therefore, an increasing incidence of CCADs in the context of stable SAH admissions may also be indicative of a larger proportion of traumatic or spontaneous CCADs in admitted patients.

Similar to the limitations of previous studies, ${ }^{41}$ etiological or causal relationships between variables and outcomes cannot be entirely assumed here as our study was purely observational. ICD-9-CM codes have historically been of questionable accuracy, particularly as they may change over time. The accuracy of the ICD-9-CM codes used in our study has been validated in previous studies, with an estimated $94 \%$ probability of identifying true cases of SAH. ${ }^{42}$

We may have also potentially missed cases of SAH not evaluated at an acute care hospital. There may also have been inaccuracies, such as the attribution of the cause of death to SAH on the basis of crude mortality data, rather than direct data from death certificates or autopsies. This analysis did not address longer term or overall SAH related mortality. Further, data on the disposition of patients at discharge may have been influenced by the overall increase in the use of and transfers to long term healthcare facilities in the USA predicated on the recent changes in insurance reimbursement and hospital overcrowding. We were also unable to examine or link mortality data beyond the hospital admission. However, in support of our results, the demographic characteristics of this group of SAH admissions were similar to those that have been identified in previous studies of SAH. ${ }^{43-45}$ Similarly, institutional underrepresentation of CCADs will decrease the incidence of observed CCADs reported to the NIS. This becomes more manifest in asymptomatic cases as the lack of clinical deficits in some instances decreases the clinical suspicion of CCAD for various operators. It is also possible that patients who may present with identified CCADs may not be reported due also to a benign clinical course, or if these patients were already being sufficiently medically treated.

Additionally, our data are limited by the quality of the NIS repository, but the advantages of our robust sample size are potentially offset by our inability to audit the data elements. The NIS data do not include important outcomes after hospitalizations classically used in research of SAH study groups. Despite these limitations, the NIS database is beneficial because 
it allows investigators to estimate national trends of several variables important for understanding the behavior of certain diseases. ${ }^{46}$ The NIS does not allow us to evaluate the effects of other variables that may have confounded our observed associations, such as the time of onset of SAH, additional comorbidities, severity and characteristics of SAH, rates of do not resuscitate orders, and timing of treatments.

\section{CONCLUSION}

This retrospective analysis of NIS data assessing the incidence and mortality of CCADs in atraumatic SAH admission requiring intervention is the first long term analysis on this subject to be reported in the academic literature. Our data suggested that the incidence of CCADs gradually, yet consistently, increased in this population during the study period. This could be due to better diagnostic techniques in recent years or more frequent use of endovascular methods in aneurysmal repair. In our study population, the incidence of CCADs was significantly higher in the endovascular coiling subgroup compared with the surgical clipping group. This could be due to more endovascular interventions in these patients, resulting in higher total complications or a surgical preference in cases presenting with dissection aneurysms. While the occurrence of CCADs had no measurable associations with mortality in the population we examined, there was a statistically significant increase in length of hospitalizations. This has implications on overall cost of care and other economic metrics. As technologies continue to evolve in the treatment and diagnosis of SAH, we must continually evaluate the efficacy and complication profiles of introduced therapies, such as endovascular coiling. This ensures sounds medical decision making on the part of the physician, and similarly affords patients a comprehensive understanding of risks attendant to these procedures.

Contributors KC assisted with data interpretation, manuscript writing/editing, and preparation for final submission. FR assisted with project development and data analysis. MM assisted with data analysis, reviewer responses, and data interpretations. $\mathrm{LB}$ assisted with data interpretation and manuscript writing. BD assisted with manuscript writing. MR assisted with manuscript editing and proofreading. AS was the principal investigator and project designer.

\section{Competing interests None.}

Provenance and peer review Not commissioned; externally peer reviewed.

\section{REFERENCES}

1 Thomas LC, Rivett DA, Attia JR, et al. Risk factors and clinical features of craniocervical arterial dissection. Man Ther 2011;16:351-6.

2 Ahmad HA, Gerraty RP, Davis SM, et al. Cervicocerebral artery dissections. Emerg Med J 1999;16:422-4.

3 Awad N, Choudry R, Durinka J, et al. Importance of technical preparation of intraarterial shunts to prevent iatrogenic arterial injury during urgent procedures. Vasc Endovascular Surg 2013;47:106-8.

4 Beletsky V, Norris JW. Spontaneous dissection of the carotid and vertebral arteries. N Engl J Med 2001;345:467.

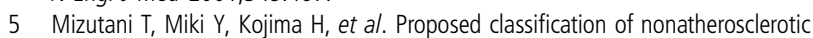
cerebral fusiform and dissecting aneurysms. Neurosurgery 1999;45:253-9; discussion 59-60.

6 Paramasivam S, Leesch W, Fifi J, et al. latrogenic dissection during neurointerventional procedures: a retrospective analysis. J Neurointervent Surg 2012;4:331-5.

7 Cloft HJ, Jensen ME, Kallmes DF, et al. Arterial dissections complicating cerebral angiography and cerebrovascular interventions. AJNR Am J Neuroradiol 2000;21:541-5.

8 Schulte S, Donas KP, Pitoulias GA, et al. Endovascular treatment of iatrogenic and traumatic carotid artery dissection. Cardiovasc Intervent Radiol 2008;31:870-4.

9 Oelerich M, Stogbauer F, Kurlemann G, et al. Craniocervical artery dissection: MR imaging and MR angiographic findings. Eur Radiol 1999;9:1385-91.

10 Thomas LC, Rivett DA, Attia JR, et al. Risk factors and clinical presentation of craniocervical arterial dissection: a prospective study. BMC Musculoskelet Disord 2012;13:164.
11 Rodallec MH, Marteau V, Gerber S, et al. Craniocervical arterial dissection: spectrum of imaging findings and differential diagnosis. Radiographics 2008;28:1711-28.

12 Gottesman RF, Sharma P, Robinson KA, et al. Clinical characteristics of symptomatic vertebral artery dissection: a systematic review. Neurologist 2012;18:245-54.

13 Wardlaw JM, White PM. The detection and management of unruptured intracranial aneurysms. Brain 2000;123:205-21.

14 Earnest Ft, Forbes G, Sandok BA, et al. Complications of cerebral angiography: prospective assessment of risk. AJR Am J Roentgenol 1984;142:247-53.

15 Heiserman JE, Dean BL, Hodak JA, et al. Neurologic complications of cerebral angiography. AJNR Am J Neuroradiol 1994;15:1401-7.

16 Theron J, Guimaraens L, Coskun 0, et al. Complications of carotid angioplasty and stenting. Neurosurg Focus 1998;5:e4.

17 Hoh BL, Chi YY, Lawson MF, et al. Length of stay and total hospital charges of clipping versus coiling for ruptured and unruptured adult cerebral aneurysms in the Nationwide Inpatient Sample database 2002 to 2006. Stroke 2010;41:337-42.

18 Hoh BL, Chi YY, Dermott MA, et al. The effect of coiling versus clipping of ruptured and unruptured cerebral aneurysms on length of stay, hospital cost, hospital reimbursement, and surgeon reimbursement at the university of Florida. Neurosurgery 2009;64(4):614-19.

19 Higashida RT, Lahue BJ, Torbey MT, et al. Treatment of unruptured intracranial aneurysms: a nationwide assessment of effectiveness. AJNR Am J Neuroradiol 2007;28:146-51.

20 Zacharia BE, Ducruet AF, Hickman ZL, et al. Technological advances in the management of unruptured intracranial aneurysms fail to improve outcome in New York state. Stroke 2011;42:2844-9.

21 Smith GA, Dagostino P, Maltenfort MG, et al. Geographic variation and regional trends in adoption of endovascular techniques for cerebral aneurysms. J Neurosurg 2011:114:1768-77.

22 Brinjikji W, Lanzino G, Rabinstein AA, et al. Age-related trends in the treatment and outcomes of ruptured cerebral aneurysms: a study of the nationwide inpatient sample 2001-2009. AJNR Am J Neuroradiol 2013;34:1022-7.

23 Fifi JT, Meyers PM, Lavine SD, et al. Complications of modern diagnostic cerebral angiography in an academic medical center. J Vasc Interv Radiol 2009;20:442-7.

24 Bendszus $M$, Koltzenburg M, Burger $R$, et al. Silent embolism in diagnostic cerebral angiography and neurointerventional procedures: a prospective study. Lancet 1999;354:1594-7.

25 Vitek JJ. Femoro-cerebral angiography: analysis of 2,000 consecutive examinations, special emphasis on carotid arteries catheterization in older patients. AJR Am J Roentgenol 1973;118:633-47.

26 Huckman MS, Shenk GI, Neems RL, et al. Transfemoral cerebral arteriography versus direct precutaneous carotid and brachial arteriography: a comparison of complication rates. Radiology 1979;132:93-7.

27 Olivecrona H. Complications of cerebral angiography. Neuroradiology 1977;14:175-81.

28 Kaku Y, Watarai H, Kokuzawa J, et al. Treatment of cerebral aneurysms: surgical clipping and coil embolization. Intervent Neuroradiol 2007;13(Suppl 1):68-72.

29 Darsaut TE, Kotowski M, Raymond J. How to choose clipping versus coiling in treating intracranial aneurysms. Neurochirurgie 2012;58:61-75.

30 Pride GL Jr, Replogle RE, Rappard G, et al. Stent-coil treatment of a distal internal carotid artery dissecting pseudoaneurysm on a redundant loop by use of a flexible, dedicated nitinol intracranial stent. AJNR Am J Neuroradiol 2004;25:333-7.

31 Matsuura JH, Rosenthal D, Jerius $\mathrm{H}$, et al. Traumatic carotid artery dissection and pseudoaneurysm treated with endovascular coils and stent. J Endovasc Surg 1997:4:339-43.

32 Mohammadian R, Taheraghdam AA, Sharifipour E, et al. Endovascular treatment of intracranial artery dissection: clinical and angiographic follow-up. Neurol Res Int 2013;2013:968380.

33 losif C, Clarencon F, Di Maria F, et al. Combined Angio-Seal and stenting rescue treatment in a case of iatrogenic common carotid artery dissection during direct puncture for ruptured intracranial aneurysm embolization: a technical note. J Neuroradiol 2013:40:130-3.

34 Dabus G, Lin E, Linfante I. Endovascular treatment of fusiform intracranial vertebral artery aneurysms using reconstructive techniques. J Neurointervent Surg 2014:6:589-94.

35 Pham MH, Rahme RJ, Arnaout 0, et al. Endovascular stenting of extracranial carotid and vertebral artery dissections: a systematic review of the literature. Neurosurgery 2011;68:856-66.

36 Lyrer $\mathrm{P}$, Engelter $\mathrm{S}$. Antithrombotic drugs for carotid artery dissection. Cochrane Database Syst Rev 2010;10:CD000255.

37 Alom J, Matias-Guiu J, Padro L, et al. Spontaneous dissection of intracranial vertebral artery: clinical recovery with conservative treatment. J Neurol Neurosurg Psychiatry 1986;49:599-600.

38 Joseph G, Raghuram L, Alexander M. Superimposition of two Wallstents to resolve iatrogenic dissection of an internal carotid artery loop. Indian Heart J 2000;52:335-8.

39 Engelter ST, Brandt T, Debette $\mathrm{S}$, et al. Antiplatelets versus anticoagulation in cervical artery dissection. Stroke 2007;38:2605-11. 
40 Anson J, Crowell RM. Cervicocranial arterial dissection. Neurosurgery 1991;29:89-96.

41 Rincon F, Rossenwasser RH, Dumont A. The epidemiology of admissions of nontraumatic subarachnoid hemorrhage in the United States. Neurosurgery 2013;73:217-22.

42 Tirschwell DL, Longstreth WT Jr. Validating administrative data in stroke research. Stroke 2002;33:2465-70.

43 Claassen J, Vu A, Kreiter KT, et al. Effect of acute physiologic derangements on outcome after subarachnoid hemorrhage. Crit Care Med 2004:32:832-8.
44 Linn FH, Rinkel GJ, Algra A, et al. Incidence of subarachnoid hemorrhage: role of region, year, and rate of computed tomography: a meta-analysis. Stroke 1996;27:625-9.

45 Claassen J, Bateman BT, Willey JZ, et al. Generalized convulsive status epilepticus after nontraumatic subarachnoid hemorrhage: the nationwide inpatient sample. Neurosurgery 2007;61:60-4.

46 Birkmeyer JD, Siewers AE, Finlayson EV, et al. Hospital volume and surgical mortality in the United States. N Engl I Med 2002:346:1128-37.

47 Martin GS, Mannino DM, Eaton S, et al. The epidemiology of sepsis in the United States from 1979 through 2000. N Engl J Med 2003;348:1546-54. 